\title{
Systemic antifungal therapy for proven or suspected invasive candidiasis: the AmarCAND 2 study
}

\author{
Olivier Leroy ${ }^{1 *}$, Sébastien Bailly², Jean-Pierre Gangneux ${ }^{3}$, Jean-Paul Mira ${ }^{4}$, Patrick Devos ${ }^{5}$, Hervé Dupont ${ }^{6}$, \\ Philippe Montravers ${ }^{7}$, Pierre-François Perrigault ${ }^{8}$, Jean-Michel Constantin ${ }^{9}$, Didier Guillemot ${ }^{10}$, Elie Azoulay ${ }^{11}$, \\ Olivier Lortholary ${ }^{12}$, Caroline Bensoussan ${ }^{13}$, Jean-François Timsit ${ }^{14}$ and AmarCAND2 study group
}

\begin{abstract}
Background: In the context of recent guidelines on invasive candidiasis (IC), how French intensive care units (ICUs) are managing IC?

Methods: This is a prospective observational multicenter cohort study. During 1 year (2012-2013), 87 French ICUs enrolled consecutive patients with suspected or proven IC (SIC or PIC) and receiving systemic antifungal therapy (SAT). Data were collected up to 28 days after inclusion.

Results: We studied 835 patients, 291 with PIC and 544 with SIC. At SAT initiation, patients with SIC were significantly more severe (SAPS II $50.1 \pm 18.7$ vs. $46.2 \pm 18.0$ ). Severe sepsis or septic shock prompted to initiate empiric SAT in $70 \%$ of SIC. Within 4 days in median, the initial SAT was modified in $49 \%$ of patients with PIC vs. $33 \%$ patients with SIC. Modifications were most often motivated by mycological results, and de-escalation was the most frequent change. Regarding compliance to IC management guidelines, echinocandin was used for 182 (62.5\%) patients with PIC, and 287 (52.7\%) of those with SIC; central venous catheter was removed in 87 (54.3\%) of patients with candidaemia, and 43 of the remaining patients received echinocandin; and de-escalation was undertaken after 5 days of SAT in 142 patients, after 10 days in 13 patients. As $20.6 \%$ of SIC were secondarily documented, 403/835 (48\%) patients had finally a proven IC. Candida albicans was the main pathogen (65.3\%), then Candida glabrata (15.9\%). The 28-day mortality rates were $40.0 \%$ in candidaemia, $25.4 \%$ in $\mathrm{CIAI}$, and $26.7 \%$ in deep-seated candidiasis. In the overall population of patients with proven IC, four independent prognostic factors were identified: immunosuppression (Odds Ratio (OR) $=1.977: 1.03-3.79495 \%$ confidence interval $(\mathrm{Cl}), \mathrm{p}=0.04)$, age $(\mathrm{OR}=1.035 ; 1.017-1.05395 \% \mathrm{Cl} ; p<0.001)$, SAPS $>46$ on ICU admission (OR $=2.894$; $1.81-4.62695 \% \mathrm{Cl} ; p<0.001)$, and surgery just before or during ICU stay (OR $=0.473 ; 0.29-0.7795 \% \mathrm{Cl} ; p<0.001)$.
\end{abstract}

Conclusion: When SAT is initiated in French ICUs, the IC is ultimately proven for $48 \%$ of patients. Empiric SAT is initiated in severely ill ICU patients. The initial SAT is often adapted, with de-escalation to fluconazole when possible. Mortality rate remains high.

Keywords: Candida, Intensive care, Cohort study, Candidiasis, Candidaemia, Critically ill

\section{Background}

The management of critically ill patients in intensive care units (ICUs) has improved during the last decades yielding increased survival of patients with complex medical

\footnotetext{
*Correspondence: oleroy@ch-tourcoing.fr

${ }^{1}$ Medical ICU, Chatilliez Hospital, Tourcoing, France

Full list of author information is available at the end of the article
}

and surgical issues. Such patients are at high risk of invasive infections, including invasive candidiasis (IC) $[1,2]$. IC can involve any organ; bloodstream infections, complicated intra-abdominal infections (cIAI), and deepseated infection are the most frequently diagnosed [3, 4]. In the EUROBACT study, fungal bloodstream infections represented $7.8 \%$ of monomicrobial infections [5]. The

\section{空 Springer}

(c) 2016 Leroy et al. This article is distributed under the terms of the Creative Commons Attribution 4.0 International License (http://creativecommons.org/licenses/by/4.0/), which permits unrestricted use, distribution, and reproduction in any medium, provided you give appropriate credit to the original author(s) and the source, provide a link to the Creative Commons license, and indicate if changes were made. 
mortality rate of IC remains high. In the EPIC II study, mortality rate of candidaemia was $42.6 \%$ [2].

Diagnosing IC remains difficult, and requires often some days [3, 6, 7]; in addition, blood cultures have insufficient diagnostic accuracy $[4,8,9]$. Of note, while some studies showed a delayed administration of systemic antifungal therapy (SAT) was associated with increased mortality [10-12], others did not found any relationship between prognosis and the timing of initial therapy [13, 14]. Therefore, SAT is often initiated empirically, despite the lack of consensus on decision-making criteria $[3,15]$. Some scores based on risk factors-based predictions have been set to help clinicians [16-18]; however, their positive predictive value remains insufficient.

As per IDSA and ESCMID guidelines, an appropriate management of IC includes the administration of the appropriate SAT [3, 4]. For suspected invasive candidiasis (SIC) as for proven candidaemia, IDSA recommends to use fluconazole or an echinocandin, preferably echinocandin for more severe patients, whereas ESCMID guidelines do not modulate their recommendations according to patient severity.

This prospective study intended to describe the management of IC in French ICUs, including the epidemiology of the Candida species involved and the outcome of the patients.

\section{Methods}

\section{Study design and objectives}

AmarCAND2 is a multicenter, prospective, observational study, involving French ICUs having managed at least one IC within the past year. Most centers were involved in the previous AmarCAND study [19].

The study primarily aimed to describe the prescribing practices for SAT in ICUs. SAT could be targeted for patient with microbiologically proven invasive candidiasis (PIC), or empirically instituted for SIC. The secondary objectives included the analysis of the Candida species involved in the IC, and of the patient outcome according to the final IC diagnosis, and finally of prognostic factors associated with mortality of the overall population of patients with a definite diagnosis of IC. In addition, the level of compliance of the participating ICUs with the ESCMID guidelines (at draft stage when the study was conducted) and with the IDSA guidelines was analyzed. With respect to IDSA guidelines, the use of echinocandin for severely ill patients, an appropriate source control (e.g., central venous catheter (CVC) removal in case of candidaemia), and de-escalation for fluconazolesusceptible strains when the patient is stabilized were evaluated. Regarding ESCMID guidelines, the compliance with the following recommendations was evaluated: a preferred use of echinocandin, the removal of any CVC in case of candidaemia (if not possible, echinocandin or liposomal amphotericin B use is recommended), and deescalation to be considered after 10 days of treatment for candidaemia.

The study was designed and overseen by an independent Scientific Committee, and coordinated by a clinical research organization. Investigators enrolled patients according to the study protocol and managed them according to their own clinical judgment. The Ethics Committee of the French Intensive Care Society and the French National Committee for Data Protection and Freedom of Information approved the study. According to French regulations, such an observational study does not require patients to sign an informed consent.

\section{Patients}

Investigators enrolled adult patients requiring SAT for PIC or SIC, during their ICU stay ( $\geq 48 \mathrm{~h}$ post-admission), except patients with recent ( $<15$ days) solid organ transplant, or neutropenia (absolute neutrophil count $<1 \times 10^{12}$ cells/L), or those receiving SAT prophylactically or for a mold infection.

\section{Clinical and mycological data collection and definition}

Data were collected using an electronic case report form. At the initiation of SAT, the patient was enrolled, and data were collected (Additional file 1: Clinical data collection).

At the time of SAT initiation, patients were divided into two groups. Patients for whom the presence of a positive microbiologic specimen prompted initiation of SAT were included in the PIC group. Patients for whom only a suspicion of IC led to initiate SAT were included in the SIC group. Nevertheless, in this later group, patients may ultimately have a documented IC if appropriate samples drawn before SAT initiation yielded Candida spp.

Documented IC was defined as candidaemia, cIAI, or deep-seated candidiasis. Candidaemia was defined by the presence of $\geq 1$ positive blood culture for Candida spp. A positive microscopic examination or culture for Candida spp. from a peritoneal sample drawn during surgery or percutaneously, excluding drain samples, defined cIAI. Finally, deep-seated candidiasis was defined by a positive specimen from a deep organ or fluid usually sterile (except urine and respiratory secretions).

\section{Mycological tests}

The mycology results provided by the laboratory of each hospital enabled patients to be classified as candidaemia, cIAI, deep-seated infection, or not proven infection. Isolates were classified as susceptible, susceptible-dose dependent, or resistant to antifungals, using various 
methods and according to the manufacturers' recommendations. In France, the main marketed methods used in routine laboratories refer to CLSI interpretive categories (http://www.clsi.org). E-test method (BioMérieux, France) was used in $75 \%$ of the centers.

\section{Modification of initial antifungal treatment}

In case of initial SAT modification, the investigator was asked to record the date and reason(s) for the modification. If a new SAT was started, the antifungal agent was recorded.

\section{Patient outcome}

At the end of the SAT, the Candida infection outcome, the date of ICU discharge, and the vital status of all patients at that date and at day 28 were collected.

\section{Statistical analyses}

Statistical analyses were performed using SAS (version 9.3). Variables were expressed as mean with standard deviation for numerical variables and as frequencies and percentages for categorical variables. Groups were compared using Wilcoxon, Chi-square, or Fisher's exact tests, as appropriate, with a statistical significance threshold of 0.05 .

A stepwise logistic regression analysis was performed to identify risk factors associated with mortality on day-28, regardless of its cause. In order to identify independent risk factors for mortality, variables were included in the multivariate model if the $P$ value was $\leq 0.20$ in bivariate analysis. Adjusted Odds ratios (AOR) were computed using logistic regression analysis including the independent predictors of mortality.

All statistical analyses were performed using SAS software, V9.1.

\section{Results}

From Oct. 2012 to Oct. 2013, 87 ICUs enrolled 870 patients (Fig. 1), of whom 35 were excluded for nonrespect of inclusion criteria $(n=6)$ and for missing data $(n=29)$. So, we studied 835 patients, 291 with PIC when SAT was started and 544 for whom SAT was started for SIC. Among the 291 patients with PIC at SAT initiation, candidaemia, cIAI, and deep-seated infections were diagnosed in 141, 129, and 45 patients, respectively ( 24 had infections at multiple sites).

Characteristics of patients at the ICU admission are listed in the Additional file 1: Table E1. Characteristics at SAT initiation are described in Table 1. Centers which enrolled more than 22 patients enrolled more SIC compared to PIC; otherwise centers did not differ significantly for the rate of PIC and SIC enrolled (Additional file 1: Table E2).

In the SIC group, the clinical and microbiological features taken into account for initiating empiric SAT are reported in Additional file 1: Table E3. Of note,

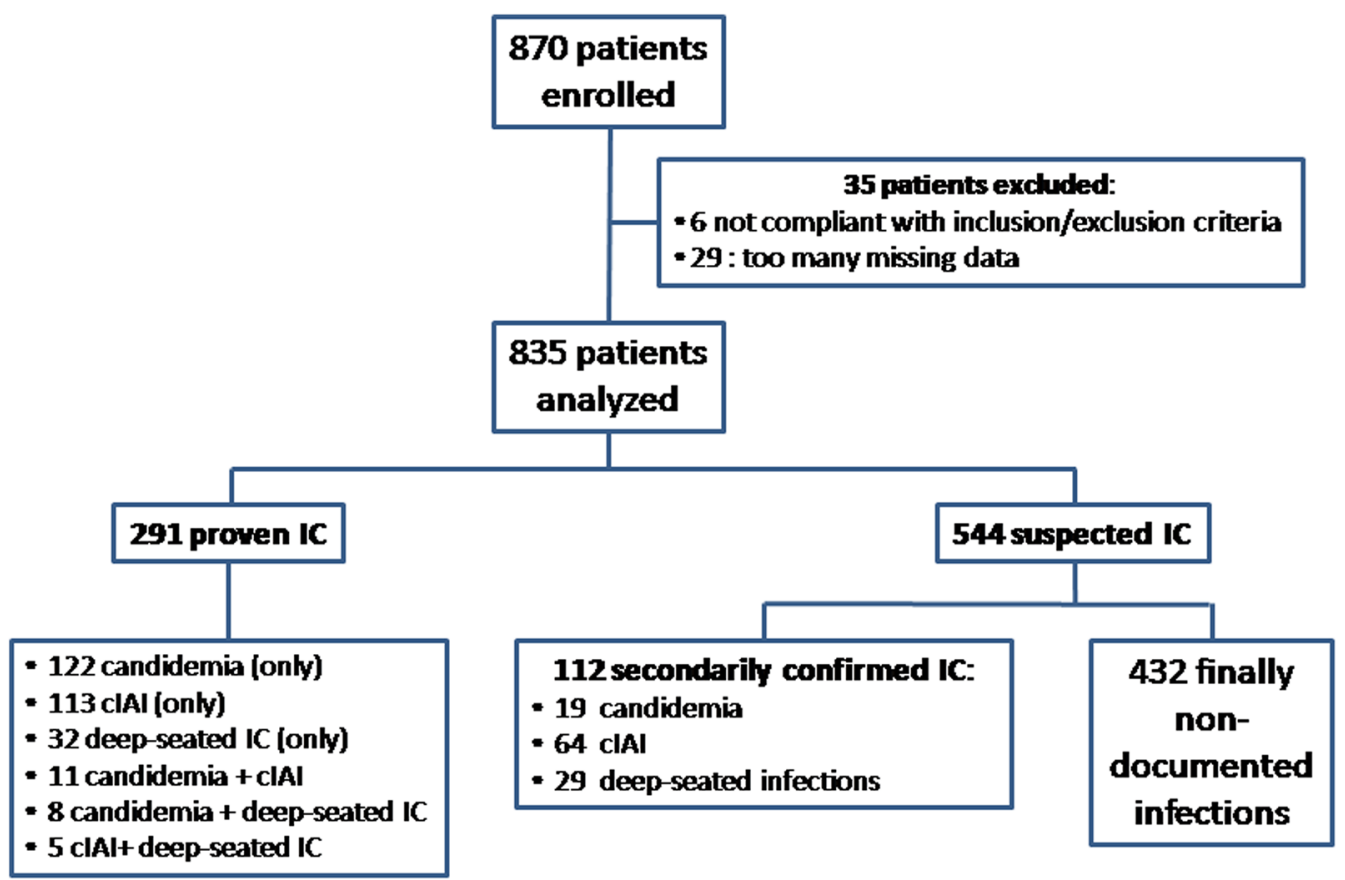

Fig. 1 Disposition of patients enrolled and analyzed. IC invasive candidiasis, CIAI complicated intra-abdominal infections 
Table 1 Characteristics of patients with initially proven and suspected invasive candidiasis

\begin{tabular}{|c|c|c|c|}
\hline Patients' characteristics & $\begin{array}{l}\text { SAT for initially proven } \\
\text { invasive candidiasis } \\
N=291\end{array}$ & $\begin{array}{l}\text { SAT for suspected } \\
\text { invasive candidiasis } \\
N=544\end{array}$ & $P$ value \\
\hline \multicolumn{4}{|l|}{ At ICU admission } \\
\hline Age (years) & $62.2 \pm 15.4$ & $61.9 \pm 14.5$ & 0.93 \\
\hline Male gender & $185(63.6)$ & $337(62.0)$ & 0.64 \\
\hline \multicolumn{4}{|l|}{ Reason for admission to ICU } \\
\hline Medical & $119(40.9)$ & $268(49.3)$ & 0.02 \\
\hline Surgical & $161(55.3)$ & $260(47.8)$ & 0.04 \\
\hline Trauma & $16(5.5)$ & $16(2.9)$ & 0.08 \\
\hline C-reactive protein (mg/L) & $165.9 \pm 109.4$ & $173.1 \pm 122.6$ & 0.67 \\
\hline Creatinine $(\mu \mathrm{mol} / \mathrm{L})$ & $145.8 \pm 109.6$ & $132.1 \pm 96.9$ & 0.13 \\
\hline Red blood cell transfusion in ICU & $163(56.0)$ & $289(53.1)$ & 0.42 \\
\hline Platelet transfusion in ICU & $60(20.6)$ & $119(21.9)$ & 0.67 \\
\hline \multicolumn{4}{|l|}{ At SAT initiation } \\
\hline Length of hospital stay before SAT (days) & $13[6 ; 23]$ & $11[4 ; 20]$ & $<0.01$ \\
\hline Length of ICU stay before SAT (days) & $5[2 ; 14]$ & $4[0 ; 11]$ & $<0.01$ \\
\hline Body temperature $\left({ }^{\circ} \mathrm{C}\right)$ & $37.6 \pm 1.3$ & $37.7 \pm 1.3$ & 0.34 \\
\hline SAPS $\|$ & $46.2 \pm 18.0$ & $50.1 \pm 18.7$ & 0.006 \\
\hline SOFA score & $7.3 \pm 4.8$ & $8.3 \pm 4.3$ & 0.0007 \\
\hline Septic shock & $120(41.2)$ & $330(66.7)$ & $<0.0001$ \\
\hline Severe sepsis & $118(40.6)$ & $203(37.3)$ & 0.36 \\
\hline \multicolumn{4}{|l|}{ Risk factors for invasive candidiasis ${ }^{\mathrm{a}}$} \\
\hline Invasive mechanical ventilation & $196(67.4)$ & $458(84.2)$ & $<0.0001$ \\
\hline Central venous catheter & $278(95.5)$ & $526(96.7)$ & 0.44 \\
\hline Urinary catheterization & $280(96.2)$ & $516(94.9)$ & 0.50 \\
\hline Antibiotic treatment & $268(92.1)$ & $481(88.4)$ & 0.12 \\
\hline Total parenteral nutrition & $149(51.2)$ & $235(43.2)$ & 0.03 \\
\hline Hemodialysis or hemodiafiltration & $92(31.6)$ & $160(29.4)$ & 0.51 \\
\hline Corticosteroid treatment & $55(18.9)$ & $151(27.8)$ & 0.005 \\
\hline Surgery just before or during ICU stay & $197(67.7)$ & $341(62.7)$ & 0.15 \\
\hline Abdominal surgery & $156(78.2)$ & $263(79.2)$ & 0.80 \\
\hline Scheduled abdominal surgery & $16(10.3)$ & $48(18.3)$ & 0.03 \\
\hline Emergency abdominal surgery & $140(89.7)$ & $215(81.7)$ & 0.03 \\
\hline Mortality on D28 & 96 (34.4\%) & $163(31.6 \%)$ & 0.42 \\
\hline
\end{tabular}

The results are given as $\mathrm{n}(\%)$ or mean $\pm \mathrm{SD}$, when indicated

SAT systemic antifungal therapy, ICU intensive care unit, SOFA sequential organ failure assessment; SAPS II: simplified acute physiology score

a Risk factors as per IDSA and ESCMID guidelines $(3,4)$

investigators reported that $70.4 \%$ patients had a septic shock, $50.9 \%$ a recent surgery, and $36.0 \%$ a persistent fever. For $54.6 \%$ of patients with empiric SAT, 4 to 8 features were reported (and from 2 to 10 features for $75 \%$ of patients); up to 14 features were reported.

The initial SAT was based on a single agent for most patients (Table 2; Additional file 1: Doses of antifungal agents). Median doses of antifungal agents are reported in additionnal file 1 . The text in additional file was as follows: At SAT initiation, the median doses of fluconazole, voriconazole, caspofungin and micafungin were $800 \mathrm{mg}$, $800 \mathrm{mg}, 70 \mathrm{mg}$ and $100 \mathrm{mg}$, respectively. On days 2 to 4 , doses were $400 \mathrm{mg}, 400 \mathrm{mg}, 50 \mathrm{mg}$ and $100 \mathrm{mg}$, respectively. The comparison of patients according to the SAT type, echinocandins vs. azoles, showed that patients receiving an echinocandin had higher severity scores both on ICU admission and at the time of SAT initiation, and a higher rate of septic shock at SAT initiation (Additional file 1: Table E4, Factors associated with echinocandin prescription).Addtional file 1. The following text could be related to this insert: A multivariate analysis was conducted to analyze the factors associated with echinocandin prescription. Variables with a p-value of 0.2 in an univariate analysis were introduced in a multivariate 
Table 2 Summary of initial antifungal treatment in patients with proven and suspected invasive candidiasis

\begin{tabular}{lll}
\hline Antifungal treatment & $\begin{array}{l}\text { SAT for proven invasive } \\
\text { candidiasis } \\
\mathbf{N = 2 9 1}\end{array}$ & $\begin{array}{l}\text { SAT for suspected invasive } \\
\text { candidiasis } \\
\text { N } \mathbf{5 4 4}\end{array}$ \\
\hline Monotherapy & $287(98.6 \%)$ & $541(99.5 \%)$ \\
Polyenes & $6(2.1 \%)$ & $6(1.1 \%)$ \\
Amphotericin B deoxycholate & 0 & 3 \\
Liposomal amphotericin B & 4 & 3 \\
Amphotericin B lipid complex & 2 & 0 \\
Azoles & $99(34.5 \%)$ & $248(45.8 \%)$ \\
Fluconazole & 99 & 244 \\
Voriconazole & 0 & 4 \\
Echinocandin & $182(63.4 \%)$ & $287(53.0 \%)$ \\
Caspofungin & 160 & 252 \\
Micafungin & 20 & 34 \\
Anidulafungin & 0 & 1 \\
Flucytosine & 0 & 0 \\
Combination therapy & 4 & 3 \\
Liposomal amphotericin B and flucytosine & 0 & 2 \\
Caspofungin and fluconazole & 2 & 0 \\
Caspofungin and voriconazole & 1 & 0 \\
Caspofungin and flucytosine & 1 & 0 \\
Amphotericin B deoxycholate and micafungin & 0 & 1 \\
\hline
\end{tabular}

The results are given as $n(\%)$

SAT systemic antifungal therapy

logistic regression. The selected variables were the followings: (1) at ICU admission: SAPSII, gender, BMI, presence of comorbidities, type of admission (medical reasons vs. other), surgery before ICU admission, interval from hospitalization; (2) at SAT initiation: parenteral nutrition, presence of bacteria, septic shock, antibacterial therapy, red blood cell transfusion, creatinine, central venous catheter, proven IC (vs. suspected). The final model showed that a proven invasive candidiasis, septic shock, SAPSII score greater than 46, ICU admission for medical reasons, a creatinine greater than $103 \mu \mathrm{mol} / \mathrm{L}$, and the presence of a central venous catheter increased significantly the probability of echinocandin prescription (see Table E4).

Overall, 325 isolates were identified among the 291 patients with PIC (Table 3). Candida albicans was the most common pathogen. Candida spp. were associated with bacterial pathogens in 19/141 patients with candidaemia and 76/129 patients with cIAI. Among the 544 patients with SIC, IC was ultimately proven for 112 for whom 128 isolates were identified (Fig. 1). Again, C. albicans was the most common pathogen (Table 3). The comparison of patients with initially proven IC $(n=291)$ vs. patients with ultimately proven IC $(n=112)$ shows the following significant differences (Additional file 1: Table E5): on ICU admission, patients with initially proven IC had a higher SOFA score; at SAT initiation, patients with ultimately proven IC were more often mechanically ventilated, had undergone more often surgery before or during ICU stay, and exhibited more often septic shock.

In the overall population, IC was thus finally proven for 403/835 patients (Fig. 1). Among patients with deepseated IC $(n=74)$, infections mainly involved pleura $(n=22)$, hepato-biliary-pancreatic tract $(n=14)$, bone or joints $(n=4)$, skin and soft tissues $(n=4)$, mediastinal space $(n=4)$, and heart valve or vascular prosthesis $(n=3)$. The comparison of patients with proven IC $(n=403)$ with patients without IC $(n=432)$ is reported in Additional file 1: Table E6. At ICU admission, patients with IC exhibited a significant lower SAPS II. At SAT initiation, patients with IC exhibited a lower SOFA score, and had less often septic shock, invasive mechanical ventilation, and previous corticosteroid treatment, whereas surgery just before or during ICU stay was more often present.

The median time elapsed between sample draw and mycological results was 1 day for the positivity to yeasts, 3-4 days for the species identification, and 5-6 days for the antifungal susceptibility. Local laboratory MIC50 (number of isolates; IQR) for the main antifungals tested was as follows: fluconazole, $0.25 \mathrm{mg} / \mathrm{L}(n=283$; $0.125-$ $1.50)$, voriconazole, $0.02 \mathrm{mg} / \mathrm{L}(n=228 ; 0.01-0.06)$, 
Table 3 Results of mycological cultures of samples from the AmarCAND2 patients, according to their status at SAT initiation: invasive candidiasis either proven, or suspected

\begin{tabular}{llll}
\hline & $\begin{array}{l}\text { SAT for proven } \\
\text { invasive candidiasis } \\
\mathbf{N = 2 9 1}\end{array}$ & $\begin{array}{l}\text { SAT for suspected } \\
\text { invasive candidiasis } \\
\mathbf{N = 5 4 4}\end{array}$ & $\begin{array}{l}\text { Overall population } \\
\mathbf{N = 8 3 5}\end{array}$ \\
\hline $\begin{array}{l}\text { Patients with at least an isolate } \\
\text { identified, i.e., proven IC }\end{array}$ & $291(100)$ & $112(20.6)$ & $403(48.3 \%)$ \\
Number of identified isolates & 325 & 128 & 453 \\
C. albicans & $206(63.4)$ & $90(70.3)$ & $296(65.3)$ \\
C. glabrata & $54(16.6)$ & $18(14.0)$ & $72(15.9)$ \\
C.parapsilosis & $19(5.8)$ & $2(1.6)$ & $21(4.6)$ \\
C. tropicalis & $14(4.3)$ & $6(4.7)$ & $20(4.4)$ \\
C. krusei & 10 & 3 & 13 \\
C. lusitaniae & 9 & 1 & 10 \\
C. kefyr & 6 & 2 & 8 \\
C. guilliermondii & 3 & 1 & 4 \\
C. dubliniensis & 2 & 0 & 2 \\
C. norvengiensis & 1 & 1 & 2 \\
C. pulcherina & 1 & 0 & 1 \\
Other & 0 & 4 & 4 \\
\hline
\end{tabular}

The results are given as $n$ (\%)

SAT systemic antifungal therapy, IC invasive candidiasis

caspofungin, $0.09 \mathrm{mg} / \mathrm{L}(n=253 ; 0.05-0.125)$, and micafungin, $0.02 \mathrm{mg} / \mathrm{L}(n=74 ; 0.01-0.03)$. The overall rate resistance among all isolates was $13 \%$ for fluconazole (including $20.3 \%$ among C. glabrata), 2 \% for voriconazole, $1.25 \%$ for micafungin, and $0.7 \%$ for caspofungin.

The initial SAT was modified for $142(48.8 \%)$ patients with PIC, $4.77 \pm 4.01$ days after initiation. The changes were motivated by mycological documentation in 118 patients. They were largely for selecting another antifungal drug (139/142). Main modifications were deescalation from an echinocandin to fluconazole for 93 patients, and escalation from fluconazole to an echinocandin for 20 patients. The initial SAT was terminated or modified for 180 (33.1\%) patients with SIC, $5.37 \pm 5.25$ days after initiation. SAT was terminated in 76 patients (confirmation of the bacterial origin of the infection) and was modified in 104, mainly according to mycological documentation. De-escalation from an echinocandin to fluconazole was performed in 60 patients, and escalation from fluconazole to an echinocandin occurred in 30.

With regard to source control, a surgical procedure $(n=191)$ or a percutaneous drainage of intra-abdominal abscess $(n=2)$ was performed in all patients with cIAI. CVC was removed in 87 (54.3\%) patients with candidaemia. This removal occurred 2 days or later after blood sampling identifying candidaemia in $34.4 \%$ of cases. Among the 73 patients without CVC removal,
6 died before the result of blood sampling identifying Candida spp. was known, and 5 had no CVC. Among the 62 remaining patients, 43 patients received an echinocandin.

Compliance to three main recommendations of IDSA guidelines was analyzed; (1) echinocandin was used for $182(62.5 \%)$ of patients with PIC, and 287 (52.7\%) of patients with SIC; (2) CVC was removed in 87 (54.3\%) patients with candidaemia; (3) de-escalation was undertaken 5 days after SAT initiation for $142(17 \%)$ patients. At least one recommendation was applied for 506 patients. With regard to the compliance to the similar recommendations of ESCMID guidelines, (1) echinocandin was used for 182 (62.5\%) of patients with PIC, and 287 (52.7 \%) of patients with SIC; (2) CVC was removed in $87(54.3 \%)$ patients with candidaemia. Among the 62 patients with CVC and mycological results known while still alive, 43 (69.3\%) were treated with echinocandin; (3) 10 days after SAT initiation, among the 484 patients still alive in ICU, de-escalation was undertaken for $13(2.7 \%)$ patients. At least one recommendation was applied for $505(60.5 \%)$ patients.

The clinical outcome of the patients according to their final diagnosis and SAT duration is depicted in Table 4. Cure rates ranged from 55 to $70 \%$. The mortality rates were $40.0 \%$ in candidaemia, $25.4 \%$ in cIAI, and $26.7 \%$ in deep-seated candidiasis. In patients with vs. without immunodeficiency, mortality rates were 58.3 vs. $37.3 \%$ 
Table 4 Clinical evolution as a function of the final diagnosis of invasive candidiasis

\begin{tabular}{|c|c|c|c|c|}
\hline & \multicolumn{4}{|l|}{ Final diagnosis } \\
\hline & $\begin{array}{l}\text { Candidaemia } \\
n=160\end{array}$ & $\begin{array}{l}\text { CIAI } \\
n=193\end{array}$ & $\begin{array}{l}\text { Deep-seated candidiasis } \\
n=74\end{array}$ & $\begin{array}{l}\text { Non-confirmed IC } \\
n=432\end{array}$ \\
\hline \multicolumn{5}{|l|}{ SOFA score at D0 } \\
\hline Mean \pm SD & $7.7 \pm 4.8$ & $7.2 \pm 4.5$ & $7.7 \pm 4.5$ & $8.4 \pm 4.3$ \\
\hline Median (min; max) & $7(0 ; 23)$ & $7(0 ; 19)$ & $7.5(0 ; 17)$ & $8(0 ; 24)$ \\
\hline \multicolumn{5}{|l|}{ SOFA score at D7 } \\
\hline Mean \pm SD & $6.9 \pm 6.0$ & $4.9 \pm 5.1$ & $6.5 \pm 5.8$ & $6.8 \pm 5.3$ \\
\hline Median (min; max) & $5(0 ; 24)$ & $3(0 ; 24)$ & $5(0 ; 21)$ & $6(0 ; 24)$ \\
\hline \multicolumn{5}{|l|}{ Clinical outcome $n(\%)$} \\
\hline Cured & $89(55.6)$ & $135(70.0)$ & $48(64.9)$ & $261(60.4)$ \\
\hline Failure & $27(16.85)$ & $20(10.35)$ & $12(16.2)$ & $42(9.7)$ \\
\hline Not determined & $33(20.6)$ & $27(14.0)$ & $13(17.6)$ & 89 (20.6) \\
\hline Information not available & $11(6.9)$ & $11(5.7)$ & $1(1.3)$ & $40(9.3)$ \\
\hline \multicolumn{5}{|l|}{ Status on D28, n (\%) } \\
\hline Alive & $90(56.3)$ & $135(70.0)$ & $53(71.6)$ & $271(62.7)$ \\
\hline Dead & $64(40.0)$ & $49(25.4)$ & $19(26.7)$ & $138(32.0)$ \\
\hline Lost to follow-up after ICU discharge & $6(3.7)$ & $9(4.6)$ & $2(2.7)$ & $23(5.3)$ \\
\hline \multicolumn{5}{|l|}{ Length of SAT among those alive (days) } \\
\hline Mean \pm SD & $24 \pm 16$ & $20 \pm 14$ & $28 \pm 25$ & $12 \pm 10$ \\
\hline Median & 20 & 17 & 21 & 10 \\
\hline
\end{tabular}

CIAI complicated intra-abdominal infections, IC invasive candidiasis, SAT systemic antifungal therapy, SD standard deviation

$(p=0.11), 40$ vs. $23.65 \%(p=0.11)$, and 30 vs. $25 \%$ $(p=0.71)$, respectively. Among patients with candidaemia, the mortality rate was $38 \%$ in patients with isolated candidaemia $(n=54 / 141), 45 \%$ in patients with candidaemia associated with cIAI $(n=5 / 11)$, and $62.5 \%$ in patients with candidaemia associated with deep-seated candidiasis $(n=5 / 8)(p=0.4)$. Among patients with candidaemia and non-immunosuppressed $(n=134)$, the mortality rate was $37.3 \%(50 / 134)$. It was not significantly different according to the removal of the CVC (35 vs. $40 \% ; p=0.59$ ), to the early SAT initiation, i.e., before the knowledge of positive blood culture (31 vs. $38 \%$; $p=0.61$ ), or to the use of echinocandin as SAT (39 vs. $31 \% ; p=0.42$ ).

In the overall population of patients with proven IC $(n=403), 121$ patients died. Factors associated with 28 -day mortality are reported in Table 5 . In multivariate analysis, four independent prognostic factors were identified: immunosuppression (Odds Ratio (OR) $=1.977$ : 1.03-3.794 $95 \%$ confidence interval (CI), $p=0.04)$, age $(\mathrm{OR}=1.035 ; 1.017-1.05395 \% \mathrm{CI} ; p<0.001)$, SAPS $>46$ on ICU admission ( $\mathrm{OR}=2.894 ; 1.81-4.62695 \% \mathrm{CI}$; $p<0.001)$, and surgery just before or during ICU stay (OR $=0.473 ; 0.29-0.7795 \% \mathrm{CI} ; p<0.001)$. Of note, early SAT instituted before the knowledge of positive sample did not influence outcome $(\mathrm{OR}=1.507$ : 0.891-2.548 $95 \% \mathrm{CI} ; p=0.13)$.

\section{Discussion}

This study on SAT in French ICUs enrolled 291 patients with PIC and 544 with SIC. Patients receiving SAT for SIC were more severely ill than those having SAT for PIC: they had higher severity scores and more often septic shock. They had more often known IC risk factors. Initial SAT was mainly based on echinocandins. However, in case of adaptation, fluconazole was mainly chosen. Mortality remains high, particularly for candidaemia (40.0\%).

The appropriate IC management includes the administration of the right antifungal agent [3, 4]. Regarding comparable population set within the previous AmarCAND study, caspofungin was administered to $18.1 \%$ of patients, while $72.7 \%$ received fluconazole [19]. In contrast, at SAT initiation for AmarCAND2, caspofungin was the most frequent agent administered. It was significantly preferred for more severe patients, for targeted as for empiric SAT. ESCMID guidelines recommend treating patients with suspected IC with echinocandin [3], similarly to IDSA guidelines [4], although the latter recommends using echinocandin for severely ill patients. Our study patients were admitted in ICU, thus severely ill, which is consistent with the rather high rate of patients treated with echinocandin $(63.4 \%$ of PIC patients, $53.0 \%$ of SIC patients). Indeed, echinocandin were significantly more often administered in case of illness severity such as septic shock or high severity scores. 
Table 5 Factors associated with 28-day mortality in patients with proven IC

\begin{tabular}{|c|c|c|c|}
\hline Patients' characteristics & Survivors $(N=273)$ & Non survivors $(N=130)$ & $P$ value \\
\hline \multicolumn{4}{|l|}{ At ICU admission } \\
\hline Age & $61.5[51 ; 70.1]$ & $66.5[57.9 ; 78.8]$ & $<.01$ \\
\hline Male gender & $171(62.6)$ & $83(63.8)$ & 0.81 \\
\hline SAPSII & $42[30 ; 52]$ & $56[45 ; 67]$ & $<0.01$ \\
\hline SOFA score & $8[5 ; 10]$ & $9.5[7 ; 13]$ & $<0.01$ \\
\hline Immunosuppression & $29(10.6)$ & $31(23.8)$ & $<0.01$ \\
\hline Reason for admission to ICU & & & $<0.01$ \\
\hline Medical & $88(32.2)$ & $66(50.8)$ & \\
\hline Elective surgical & $24(8.8)$ & $14(10.8)$ & \\
\hline Emergency surgical & $144(52.7)$ & $48(36.9)$ & \\
\hline Trauma & $17(6.2)$ & $2(1.5)$ & \\
\hline Red blood cell transfusion in ICU & $141(51.6)$ & $79(60.8)$ & 0.09 \\
\hline Platelet transfusion in ICU & $49(17.9)$ & $36(27.7)$ & 0.03 \\
\hline \multicolumn{4}{|l|}{ At SAT initiation } \\
\hline Body temperature $\left({ }^{\circ} \mathrm{C}\right)$ & $38[37 ; 38.5]$ & $37.6[36.9 ; 38.2]$ & $<0.01$ \\
\hline SOFA score & $6[3 ; 9]$ & $9[6 ; 13]$ & $<0.01$ \\
\hline Septic shock & $110(40.3)$ & $72(55.4)$ & $<0.01$ \\
\hline Severe sepsis & $117(42.9)$ & $48(36.9)$ & 0.26 \\
\hline Invasive mechanical ventilation & $191(70)$ & $97(74.6)$ & 0.33 \\
\hline Central venous catheter & $261(95.6)$ & $126(96.9)$ & 0.53 \\
\hline Urinary catheterization & $259(94.9)$ & $129(99.2)$ & 0.03 \\
\hline Hemodialysis or hemodiafiltration & $68(24.9)$ & $53(40.8)$ & $<0.01$ \\
\hline Total parenteral nutrition & $135(49.5)$ & $64(49.2)$ & 0.97 \\
\hline Corticosteroid treatment & $44(16.1)$ & $38(29.2)$ & $<0.01$ \\
\hline Surgery just before or during ICU stay & 209 (76.6) & $75(57.7)$ & $<0.01$ \\
\hline Initial SAT & & & 0.26 \\
\hline Amphotericin B & $4(1.5)$ & $2(1.5)$ & \\
\hline Fluconazole & $112(41)$ & $40(30.8)$ & \\
\hline Voriconazole & $2(0.7)$ & $1(0.8)$ & \\
\hline Echinocandins & $155(56.8)$ & $87(66.9)$ & \\
\hline SAT instituted before the knowledge of positive sample & & & 0.14 \\
\hline No & $191(70)$ & $100(76.9)$ & \\
\hline Yes & $82(30)$ & $30(23.1)$ & \\
\hline
\end{tabular}

The results are given as $n(\%)$ or median (min; max), when indicated

SAT systemic antifungal therapy, ICU intensive care unit, SOFA sequential organ failure assessment, SAPS // simplified acute physiology score

Another recommendation of both guidelines which was evaluated relates to a careful control of source. Most, but not all, patients with candidaemia had CVC, which was removed in one patient out of two. This rate is not very high; however, IDSA and ESCMID recommendations differ slightly. IDSA guidelines recommend CVC removal, whereas ESCMID guidelines add that patients whom CVC cannot be removed should be treated with echinocandin or liposomal amphotericin B. Indeed, among patients with candidaemia and CVC not removed, most patients $(69.3 \%)$ received echinocandin. For patients with cIAI, the source control is abdominal surgery or at least peritoneal drainage, which was undertaken for all, as it was a study inclusion criterion. The last recommendation of both guidelines which was evaluated relates to the de-escalation of SAT. According to IDSA guidelines, de-escalation should be performed in case of IC due to fluconazole-susceptible strains when the patient becomes stable, whereas ESCMID guidelines recommend de-escalation after ten days of intravenous SAT for patients with candidaemia. Overall, regarding de-escalation, IDSA recommendation was applied for $17 \%$ of all patients. The compliance rate with IDSA or ESCMID guidelines is rather poor. However, although ESCMID guidelines were circulated through 2011 ECCMID workshop slides, they were officially issued after the study start. Regarding the 
duration of SAT, it was shorter among patients whose IC was eventually not documented than among patients with proven IC, and similar to that observed in the FongiDay study: 11.7 days on average and no longer than 16 days [20].

In our study, C. albicans remained the leading Candida species, followed by C. glabrata, which is the leading non-albicans pathogen in France [19, 21], in Northern European countries [22-24], and in the US [11, 25]. This rather low rate of $C$. glabrata, close to $16 \%$ in our study, explains why fluconazole is chosen in half of patients. This rate is comparable to that observed during the previous AmarCAND study [19]. It remained stable despite the use of echinocandins, in contrast with the results of a recent French study showing a higher rate of $C$. glabrata $(20.0 \%)$, increasing after previous exposure to caspofungin [26]. Regarding antifungal susceptibility, antifungal resistance was uncommon except for $13 \%$ of isolates classified as fluconazole-resistant.

Mycological cultures require some days for the full process from the sampling to the susceptibility results. Mycological results were the main drivers for treatment modifications, which occurred in almost half of the targeted SATs. Empiric SAT was modified in one-third of patient, more often based on mycological results than on clinical changes. In $42 \%$ patients whom treatment was modified, the SAT was terminated based on positive bacteriological results combined with negative mycological tests results. For empiric as for targeted SAT, the most frequent initial drug was caspofungin, and the most frequent agent modification was de-escalation to fluconazole. The delayed availability of mycological results likely explains the high rate of patients with empiric SAT [27]. Other laboratory tests, not based on cultures, have been developed, such as Candida-specific polymerase-chainreaction, or tests based on the detection of yeasts antigens, or of antibodies directed against these antigens. In our study, the use of non-culture-based diagnostic methods to suspect IC and so to begin SAT was still anecdotal (less than $4 \%$ ). These tests are now recommended by the ESCMID guidelines for the IC diagnosis [8], and will probably be increasingly implemented as far as robust and standardized methods will be available.

The identification of high-risk patients who may benefit from empiric SAT is still challenging. Major risk factors for IC are well known [3, 4, 7, 28]; however, they are so common in ICU patients than other tools are needed. The Candida colonization index and the Candida score are useful for their good negative predictive value enabling to identify patients at low risk of IC [16, 18]. Whether the study investigators used these scores was not captured. Nevertheless, it could be suggested that, in this study, the overall conditions leading investigators to initiate empiric SAT had both low specificity since a minority of patients with empiric SAT had a secondarily proven IC (112/544) and low sensitivity since only a minority of patients with proven IC benefited from an early SAT (19/141 candidaemia, 64/129 cIAI, and 29/45 deep-seated infections). These rates are disappointing compared to results showed by Bruyere et al. in a study on ICU patients with suspected IC based on uncontrolled sepsis despite at least one 2-day course of antibacterial agents: the use of the Candida score with a cut-off point at 3 or more triggering SAT prescription for these patients was associated with sensitivity, specificity, positive and negative value of $69.2,82.1,69.2$, and $82.1 \%$, respectively [29]. However, the rate of secondarily proven IC among those empirically treated (20.4\%) likely reflects the specific interest of participating ICUs regarding invasive candidiasis risk assessment.

IC remains associated with a high mortality rate. In our study, we observed a day- 28 mortality rate set at $40,25.7$, and $25.4 \%$ for candidaemia, deep-seated IC, and cIAI, respectively. Such a result is in accordance with literature data. In a nationwide US study collecting data on bloodstream infections from 1995 to 2002, the crude mortality rate of candidaemia among ICU patients reached $47 \%$ [30]. In AmarCAND1 study, the 30-day mortality was $45.9 \%$ in patients with PIC [19]. Finally, in a study conducted from 2002 to 2010 among ICU patients with candidaemia, the day- 28 mortality rate increased from 41.5 to $56.9 \%$ [26]. Among risk factors of poor prognosis identified in this study, a greater age, immunodeficiency, and SAPSII $>46$ are well-known risk factors of poor prognosis in ICU patients overall. A recent surgery was not previously identified as a protective factor for IC prognosis. However, the surgical origin of ICU patients was already identified as a factor of good prognosis for candidaemia [31].

This study had some limitations. First, it was conducted in one country and results could not fully be applied to other countries. However, it involved a large number of ICUs with a broad case-mix range of medical and surgical patients, and provides an accurate picture of the SAT practices. Second, it was an observational study, without any study-specific intervention; however, it is the first study comparing the management of PIC and SIC within the same establishments. Third, microbiological methods used to determine MIC were different in the participant hospitals, leading to interpret these results with caution. Finally, fungal infection management was likely a field of interest for most investigators; therefore, physicians were more likely to know and follow the guidelines and to administrate properly the SAT. In addition, we cannot ensure that absolutely all consecutive patients receiving SAT were included in the study. Therefore, it might 
hamper the extrapolation of these study results to other ICUs.

\section{Conclusion}

This large prospective observational study conducted showed the management of IC and the strategy of SAT use in French ICUs. Almost half of patients had an ultimately proven IC, of whom only $27 \%$ received early SAT before mycological documentation. This later point could explain why the mortality associated to IC remained high, particularly in patients with candidaemia. ESCMID guidelines were not officially issued at the time our study was conducted, and there are some discrepancies between the IDSA and the ESCMID recommendations we evaluated, which altogether might have contributed to the rather low rate of compliance observed in this study. Comparatively to AmarCAND1, the diversity of the Candida species was maintained, the rate of IC due to $C$. albicans remained high, and the rate of C. glabrata remained stable. Further analyses will be conducted to further describe epidemiological and mycological study results.

\section{Additional file}

Additional file 1. Table E1. Characteristics of patients with proven and suspected invasive candidiasis at ICU admission. Table E2. Rate of proven versus suspected invasive candidiasis enrolled by center according to center characteristics. Table E3. Among the 544 ICU patients with SAT for suspicion of invasive candidiasis, characteristics of patient reported as having triggered the decision to initiate a systemic antifungal therapy. Table E4. Factors in favor of the prescription of echinocandin agent at SAT initiation (multivariate analysis). Table E5. Comparison of patients with initially vs. ultimately proven invasive candidiasis. Table E6. Comparison of patients with invasive candidiasis vs. without invasive candidiasis.

\section{Authors' contributions}

$\mathrm{OL}$, JPG, JPM, HD, PM, PFP, JMC, DG, EA, OL, CB and JFT designed the study. $\mathrm{SB}$ and PD analyzed the data. OL, SB, JPM, PM and JFT wrote the article. All authors read and approved the final manuscript.

\footnotetext{
Author details

${ }^{1}$ Medical ICU, Chatilliez Hospital, Tourcoing, France. ${ }^{2}$ U823, Grenoble 1 University, La Tronche, France. ${ }^{3}$ Mycology, Rennes University Hospital, Rennes, France. ${ }^{4}$ Medical ICU, Cochin University Hospital, Paris, France. ${ }^{5}$ Bio Statistics Unit, Lille University Hospital, Lille, France. ${ }^{6}$ Surgical ICU, Amiens University Hospital, Amiens, France. ${ }^{7}$ Anesthesiology and Critical Care Medicine, Bichat-Claude Bernard University Hospital, Paris, France. ${ }^{8}$ Medical-surgical ICU, Montpellier University Hospital, Montpellier, France. ${ }^{9}$ Perioperative Medicine Department, Clermont-Ferrand University Hospital, Clermont-Ferrand, France. 10 Unité de Pharmaco-épidémiologie et Maladies Infectieuses, Institut Pasteur, Paris, France. ${ }^{11}$ Medical ICU, Saint-Louis University Hospital, Paris, France. ${ }^{12}$ Necker Pasteur Center for Infectious Diseases, Necker Enfants-Malades Hospital, Paris, France. ${ }^{13}$ Medical Affairs, MSD France, Courbevoie, France. ${ }^{14}$ Medical ICU, Bichat-Claude Bernard University Hospital, Paris, France.
}

\section{Acknowledgements}

Members of the independent Scientific Committee: Olivier Leroy, Jean-François Timsit, Jean-Pierre Gangneux, Elie Azoulay, Jean-Michel Constantin, Hervé Dupont, Olivier Lortholary, Jean-Paul Mira, Philippe Montravers, Pierre-François
Perrigault. The authors thank Celine Feger, M.D., (EMIBiotech), who provided assistance in preparing and editing the manuscript. Some results of this study were presented during the 43ème Congrès International de la Société de Réanimation de Langue Française (Paris $21-23$ janvier 2015).

The AmarCAND2 Study Group (by alphabetical order of the ICU physician name): Drs. Aait Hssain (Clermont-Ferrand), Adda (Marseille), Allaouchiche (Lyon), Ammenouche (Amiens), Angel (Marseille), Argaud (Lyon), Badetti (Marseille), Baldesi (Aix-en-Provence), Barthet (St Gaudens), Bastien (Bron), Baudin (Paris), Bellec (Montauban), Blasco (Besançon), Bollaert (Nancy), Bonadona (Grenoble-La Tronche), Bretonnière (Nantes), Brocas (Evry), Brua (Vandoeuvre-lès-Nancy), Bruder (Marseille), Brunin (Boulogne), Cabaret (Lomme), Carpentier (Rouen), Cartier (Grenoble-La Tronche), Cerf (Suresnes), Chabanne (Clermont-Ferrand), Charles (Dijon), Cheval (Hyères), Cinotti (St Herblay), Cohen (Bobigny), Constantin (Clermont-Ferrand), Cousson (Reims), Delpierre (Lagny S/Marne), Demory (Toulon), Diconne (Saint Etienne), Du Cheyron (Caen), Dubost (St Mandé), Dumenil (Clamart), Durand (Grenoble), Duroy (Vesoul), Forel (Marseille), Foucher-Lezla (Angers), Fratea (Paris), Gally (Mulhouse), Gaudard (Montpellier), Geffe (Metz), Gergaud (Angers), Gette (Metz), Girault (Rouen), Goubaux (Nice), Gouin (Rouen), Grenot (Beuvry), Grossmith (Aubagne), Guelon (Clermont-Ferrand), Guerin-Robardey (Beauvais), Guervilly (Marseille), Hayl-Slayman (Bourgoin-Jallieu), Hilbert (Bordeaux), Houissa (Paris), Hraiech (Marseille), Ichai (Villejuif), Jung (Montpellier), Kaidomar (Fréjus), Karoubi (Bobigny), Kherchache (Agen), Lambiotte (Maubeuge), Lamhaut (Paris), Launoy (Strasbourg), Lebreton (Montpellier), Lefrant (Nîmes), Lemaire (Roubaix), Lepape (Pierre-Bénite), Lepoivre (St Herblain), Leroy (Tourcoing), Lesieur (La Rochelle), Levy (Vandoeuvre-lès-Nancy), Luyt (Paris), Mahe (Nantes), Mahul (St Etienne), Mateu (Charleville-Mézières), Megarbane (Paris), Merle (Créteil), Mira (Paris), Montcriol (Toulon), Mootien (Mulhouse), Navellou (Besançon), Ouattara (Pessac), Page (Boulogne), Perrigault (Montpellier), Petitpas (Poitiers), Plantefeve (Argenteuil), Quinart (Bordeaux), Quintard (Nice), Ragonnet (Marseille), Roquilly (Nantes), Ruiz (Toulouse), Saliba (Villejuif), Samba (Caen), Schmitt (Lyon), Seguin (Rennes), Sejourne (Dechy), Tellier (Chambray-les-Tours), Thevenot (Perpignan), Tonnelier (Brest), Van Grunderbeek (Lens), Vincent (Saintes), Wiramus (Marseille), and Zogheib (Amiens).

\section{Competing interests}

Authors had full access to the database, and the statistical analyses were conducted by an academic team: Bio statistics Unit, Lille University Hospital, Lille, France, which worked in full independence from MSD. EA has been a consultant to Astellas, Alexion, Cubist, Gilead and MSD, and has benefited from grants to his research unit from Gilead and Pfizer. CB is an employee of MSD France. J-MC has been a consultant to MSD. HD has been a consultant to Astellas, Gilead, Cubist, Astrazeneca, Merck and Pfizer. J-PG has been a consultant to Astellas, Gilead, Merck and Pfizer. DG has benefited from grants of the Principalty of Monaco to his research unit. OLeroy has been consultant to Astellas, Gilead, Merck, Novartis, Pfizer and Sanofi. OLortholary has been consultant to Gilead Sciences and Novartis and member of the speaker's bureau of Astellas, Basilea, Merck, Pfizer and Sanofi. J-PM has been a consultant to Astellas, Gilead, MSD, and LFB. PM has been a consultant to Astra-Zeneca, Cubist, MSD, Pfizer, and TMC. P-FP has been a consultant to MSD and Pfizer. JFT has given lectures for symposiums set up by Astellas, Pfizer, MSD, 3 M, Novartis, and Gilead; has benefited from unrestricted research grants to his research unit from $3 \mathrm{M}$, MSD, and Astellas; and has been a consultant involved in scientific boards for MSD, 3 M, and Bayer. PD and SB have no competing interests.

\section{Funding}

This work was supported by MSD France, sponsor of the study.

Received: 6 October 2015 Accepted: 13 December 2015

Published online: 08 January 2016

\section{References}

1. Vincent JL, Rello J, Marshall J, et al. International study of the prevalence and outcomes of infection in intensive care units. JAMA. 2009;302:2323-9. 
2. Kett DH, Azoulay E, Echeverria PM, et al. Candida bloodstream infections in intensive care units: analysis of the extended prevalence of infection in intensive care unit study. Crit Care Med. 2011;39:665-70.

3. Cornely OA, Bassetti M, Calandra T, et al. ESCMID guideline for the diagnosis and management of Candida diseases 2012: non-neutropenic adult patients. Clin Microbiol Infect. 2012;18(Suppl 7):19-37.

4. Pappas PG, Kauffman CA, Andes D, et al. Clinical practice guidelines for the management of candidiasis: 2009 update by the Infectious Diseases Society of America. Clin Infect Dis. 2009;48:503-35.

5. Tabah A, Koulenti D, Laupland K, et al. Characteristics and determinants of outcome of hospital-acquired bloodstream infections in intensive care units: the EUROBACT International Cohort Study. Intensive Care Med. 2012;38:1930-45

6. Blot S, Charles PE. Fungal sepsis in the ICU: are we doing better? Trends in incidence, diagnosis, and outcome. Minerva Anestesiol. 2013;79:1396-405.

7. Leon C, Ostrosky-Zeichner L, Schuster M. What's new in the clinical and diagnostic management of invasive candidiasis in critically ill patients. Intensive Care Med. 2014;40:808-19.

8. Cuenca-Estrella M, Verweij PE, Arendrup MC, et al. ESCMID guideline for the diagnosis and management of Candida diseases 2012: diagnostic procedures. Clin Infect Dis. 2012;18(Suppl 7):9-18.

9. Nguyen MH, Wissel MC, Shields RK, et al. Performance of Candida realtime polymerase chain reaction, beta-D-glucan assay, and blood cultures in the diagnosis of invasive candidiasis. Clin Infect Dis. 2012;54:1240-8.

10. Kollef M, Micek S, Hampton N, et al. Septic shock attributed to Candida infection : importance of empiric therapy and source control. Clin Infect Dis. 2012;54:1739-46.

11. Garey KW, Rege M, Pai MP, et al. Time to initiation of fluconazole therapy impacts mortality in patients with candidemia: a multi-institutional study. Clin Infect Dis. 2006:43:25-31.

12. Morrell M, Fraser VJ, Kollef MH. Delaying the empiric treatment of Candida bloodstream infection until positive blood culture results are obtained: a potential risk factor for hospital mortality. Antimicrob Agents Chemother. 2005;49:3640-5.

13. Colombo AL, Guimaraes T, SukienikT, et al. Prognostic factors and historical trends in the epidemiology of candidemia in critically ill patients: an analysis of five multicenter studies sequentially conducted over a 9-year period. Intensive Care Med. 2014;40:1489-98.

14. Arendrup MC, Sulim S, Holm A, et al. Diagnostic issues, clinical characteristics, and outcomes for patients with fungemia. J Clin Microbiol. 2011;49:3300-8.

15. Puig-Asensio M, Padilla B, Garnacho-Montero J, et al. Epidemiology and predictive factors for early and late mortality in Candida bloodstream infections: a population-based surveillance in Spain. Clin Microbiol Infect. 2014;20:0245-54

16. Leon C, Ruiz-Santana S, Saavedra P, et al. Usefulness of the "Candida score" for discriminating between Candida colonization and invasive candidiasis in non-neutropenic critically ill patients: a prospective multicenter study. Crit Care Med. 2009;37:1624-33
17. Ostrosky-Zeichner L, Pappas PG, Shoham S, et al. Improvement of a clinical prediction rule for clinical trials on prophylaxis for invasive candidiasis in the intensive care unit. Mycoses. 2009;54:46-51.

18. Eggimann P, Pittet D. Candida colonization index and subsequent infection in critically ill surgical patients: 20 years later. Intensive Care Med. 2014;40:1429-48.

19. Leroy O, Gangneux JP, Montravers P, et al. Epidemiology, management, and risk factors for death of invasive Candida infections in critical care: a multicenter, prospective, observational study in France (2005-2006). Crit Care Med. 2009;37:1612-8

20. Azoulay E, Dupont H, Tabah A, et al. Systemic antifungal therapy in critically ill patients without invasive fungal infection. Crit Care Med. 2012;40:813-22

21. Parmeland L, Gazon M, Guerin C, et al. Candida albicans and non-Candida albicans fungemia in an institutional hospital during a decade. Med Mycol. 2013;51:33-7.

22. Arendrup MC, Dzajic E, Jensen $\mathrm{RH}$, et al. Epidemiological changes with potential implication for antifungal prescription recommendations for fungaemia: data from a nationwide fungaemia surveillance programme. Clin Microbiol Infect. 2013;19:E343-53.

23. Das I, Nightingale P, Patel M, et al. Epidemiology, clinical characteristics, and outcome of candidemia: experience in a tertiary referral center in the UK. Int J Infect Dis. 2011;15:e759-63.

24. Ericsson J, Chryssanthou E, Klingspor L, et al. Candidaemia in Sweden: a nationwide prospective observational survey. Clin Microbiol Infect. 2013;19:E218-21.

25. Pfaller MA, Andes DR, Diekema DJ, et al. Epidemiology and outcomes of invasive candidiasis due to non-albicans species of Candida in 2,496 patients: data from the Prospective Antifungal Therapy (PATH) registry 2004-2008. PLoS One. 2014;9:e101510.

26. Lortholary $\mathrm{O}$, Renaudat $\mathrm{C}$, Sitbon $\mathrm{K}$, et al. Worrisome trends in incidence and mortality of candidemia in intensive care units (Paris area, 2002-2010). Intensive Care Med. 2014;40:1303-12.

27. Paugam A, Ancelle T, Lortholary $O$, et al. Longer incubation times for yeast fungemia: importance for presumptive treatment. Diag Microbiol Infect Dis. 2014;80:119-21.

28. Guery BP, Arendrup MC, Auzinger G, et al. Management of invasive candidiasis and candidemia in adult non-neutropenic intensive care unit patients: part I Epidemiology and diagnosis. Intensive Care Med. 2009:35:55-62.

29. Bruyere R, Quenot JP, Prin S, Dalle F, Vigneron C, Aho S, et al. Empirical antifungal therapy with an echinocandin in critically-ill patients: prospective evaluation of a pragmatic Candida score-based strategy in one medical ICU. BMC Infect Dis. 2014;14:385.

30. Wisplinghoff H, Seifert H, Tallent SM, et al. Nosocomial bloodstream infections in pediatric patients in United States hospitals: epidemiology, clinical features and susceptibilities. Ped Infect Dis J. 2003;22:686-91.

31. Charles PE, Doise JM, Quenot JP, Aube H, Dalle F, Chavanet P, et al. Candidemia in critically ill patients: difference of outcome between medical and surgical patients. Intensive Care Med. 2003;29(12):2162-9.

\section{Submit your manuscript to a SpringerOpen ${ }^{\circ}$ journal and benefit from:}

- Convenient online submission

- Rigorous peer review

- Immediate publication on acceptance

- Open access: articles freely available online

- High visibility within the field

- Retaining the copyright to your article

Submit your next manuscript at $>$ springeropen.com 